\title{
Protocol
}

\section{A proposed study using psychophysiological biomarkers to evaluate the effectiveness of cat-cow yoga exercise to reduce chronic musculoskeletal low back pain}

\section{OPEN ACCESS}

Citation: Batool F, Noushad S, Ahmed S. A proposed study using psychophysiological biomarkers to evaluate the effectiveness of cat-cow yoga exercise to reduce chronic low back pain. APP. 2021;8(2): 112-119

\section{Corresponding Author Email: farahbatoo197@gmail.com}

DOI: 10.29052/2412-3188.v8.i2.2021.112119

Received 18/05/2021

Accepted 08/10/2021

Published 01/12/2021

Copyright $\odot$ The Author(s). 2021 This is an open access article distributed under the terms of the Creative Commons Attribution 4.0 International License, which permits unrestricted use, distribution, and reproduction in any medium, provided the original author and source are credited.

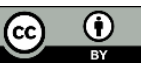

Funding: The author(s) received no specific funding for this work.

Conflicts of Interests: The authors have declared that no competing interests exist.

\author{
Syeda Farah Batool1,2 iD, Shamoon Noshad2,3 iD \& Sadaf Ahmed1,3 iD \\ ${ }^{1}$ Department of Physiology, University of Karachi, Karachi-Pakistan. \\ 2Department of Psychology, Malir University of Science \& Technology, Karachi-Pakistan. \\ 3Psychophysiology Research Lab, M.A.H.Q. Biological research center, University of Karachi, \\ Karachi-Pakistan.
}

\section{Abstract}

Background: Low Back Pain (LBP) is a painful condition of the musculoskeletal system that affects the quality of life and causes disabilities that can cease or limit daily life activities. Around $85 \%$ of the population has encountered LBP at least once in their lives. Due to sustained or improper postures, the incidence rate of LBP is reportedly high amongst healthcare providers worldwide. The treatments to manage LBP are generally some Non-steroidal anti-inflammatory drugs (NSAIDs), which only give short-term relief and are seemingly ineffective after a particular time, so higher doses are needed. This study aims to test the Cat-Cow yoga posture to manage LBP in longer terms.

Methodology: It will be a uni-center randomized control trial, and the participants with musculoskeletal low back pain will be randomly allocated into two groups. Group 1 will receive the intervention, cat-cow yoga sessions, and Group 2 will get the general care guide. Altered levels of cortisol, substance $P$ and beta-endorphins will be measured and compared at baseline and after completion of 12 weeks.

Discussion: A practical and cost-friendly intervention that can help Back pain sufferers to reduce their pain. This study will determine the efficacy of a useful and cost-effective yoga technique to overcome the psychophysiological manifestations of musculoskeletal CLBP.

\section{Keywords}

Low Back Pain, Healthcare, Nurses, Substance P, Beta-endorphins. 


\section{Introduction}

Low back pain (LBP) is a dominant reason for musculoskeletal discomfort globally ${ }^{1}$. LBP is characterized as a symptom, but in some cases, it is implied as a pathological condition with unknown etiologies depending on its severity and duration ${ }^{2}$. Low back pain is considered 'Chronic' if it persists for three months or longer than three months. Chronic low back pain (CLBP) is responsible for disability, compromised life quality, and absenteeism from work worldwide ${ }^{3}$. Approximately $70 \%$ to $85 \%$ of adults have complained about the occurrence of such pain at least once in their life span. CLBP can cause a higher number of years lost to disability than that of other morbidities like cancers, respiratory syndromes, AIDs, accidents, and childbirth complications, and it is categorized as one of the ten most potential causes that contribute to illness and disability by Global Burden of Disease (GBD) ${ }^{4}$.

It is estimated that $37 \%$ of total LBP cases are due to strenuous physical work. Many occupations are said to be riskier for getting LBP5. Sitting is a widely identified risk factor of work-related low back pain. At the same time, other work demands such as the frequent lifting of heavy stuff, intense physical work, and improper postures are also potential risk factors for LBP. In healthcare sectors, nurses are more likely to suffer from injuries and work-related musculoskeletal illnesses, such as LBP, than other healthcare workers ${ }^{5,6}$. It is evident from prior studies that psychological, social, behavioral, and demographical factors are linked with the onset of LBP. A sedentary lifestyle, smoking, and age increase the risk of LBP. Nurses' ergonomic exposures, such as the extent of direct interaction with the patient, posture, need to lift objects or attendee, work-related stress, and job satisfaction, put them at high risk of getting LBP6.

Along with the physical distress, LBP is also the reason for extended occupational leaves, and because of LBP, many healthcare professionals either switch their jobs or quit the profession 7 . The Prevalence of LBP in nurses is $71.85 \%$ in Asian countries ${ }^{8}, 82.7 \%$ in southwest Nigeria, Africa $93.53 \%$ in Saudi Arabia $^{10}$, and $69.5 \%$ in Iran ${ }^{11-13}$. In Pakistan, $65 \%$ of nurses are suffering from LBP in a study conducted in Lahore ${ }^{14}$.

There are various guidelines available for the diagnosis of non-specific low back pain. Its severity and intensity can be diagnosed by using different assessing tools or scales. Natural biological markers for pain could also be used as a diagnostic tool for the evaluation of LBP. Substance P (SP), Cortisol, and Beta Endorphins are supposed to be an effective sources of estimating pain intensity and its manifestations. Substance $\mathrm{P}$ is said to be involved in the underlying mechanism of intensifying the pain by increasing the inflammation and inflammatory markers ${ }^{15-17}$. On the contrary to this beta-endorphins, serves as an endogenous opioid and tend to relax the patient by increasing the availability of serotonin and dopamine in the synapse ${ }^{18,19}$. Kallman $^{20}$ has stated in his study that substance $\mathrm{P}$ and Beta Endorphins are not reliable pain regulatory markers in the patients' saliva of chronic neuropathic pain as according to their results, salivary substance $\mathrm{P}$ and beta-endorphins does not show much inclination ${ }^{20}$.

Specific guidelines were established by the US, UK, and other countries to treat LBP. These guidelines suggested different stages of treatment. Firstly, all guidelines emphasize that patients with non-specific LBP must learn to manage the pain independently and increase their physical 
activities with manageable breaks. If the pain persists, as the second line of care, the US and Danish guidelines highly recommend non-pharmacological treatments such as cognitive behavioral therapy and physical exercises $^{21}$. Yoga, aerobics, Tai Chi are among the highly endorsed nonpharmacological interventions to treat nonspecific chronic LBP. The use of nonsteroidal anti-inflammatory drugs (NSAIDs) and other medications to cure LBP is discouraged by physicians and scientists because of their side effects and low efficacy in reducing pain ${ }^{21,22}$.

In all the physical exercises, yoga appeared to be moderately effective in managing LBP. There are several postures of yoga that are practiced and recommended by physiotherapists and orthopedists. Still, there is very scarce data that proves the clinical significance and efficacy of yoga. A systemic review of many clinical trials suggests a moderate certainty about the potential of yoga in relieving pain ${ }^{23,24}$. This study aims to determine the effectiveness of yoga as a potential intervention in the management of LBP. Cat-cow yoga posture is selected for this purpose. There are two basic shapes your torso and spine can make: flexion and extension. In the simplest explanation, flexion is a rounded back, and extension is an arched back. Cat/Cow alternates between flexion Cat and extension Cow. This exercise will help to relax the muscles and will make them more flexible and mobile ${ }^{25}$.

\section{Methodology}

\section{Study Design}

It is a uni-center Randomized controlled trial. Subjects from diverse ethnicities, educational backgrounds, and different socioeconomic statuses are preferred for this study. Subjects will be included in the study if they meet the eligibility criteria and have experienced low back pain in the last three months. Written informed consent will be obtained from each study subject after providing detailed information regarding the objectives of the study and its duration. Subjects will be randomly assigned to either control or interventional group. Outcome measures will be assessed at the baseline and after three months.

\section{Ethical Concerns}

The study will be conducted under the declaration of Helsinki. All ethical protocols will be followed during the study, and the participants will obtain written consent. This study is consulted and approved by Ethical Committee with the approval number ERC/S20/P-001.

\section{Eligibility Criteria \\ Inclusion Criteria}

Participants must meet all the following inclusion criteria to participate in this study.

1. Age between 25 to 45 years.

Answer YES to the following questions

2. Have low back pain constantly or on most days for the last three months?

3. Have you sought care from a health care provider due to back pain?

Following scales will be used to assess the participants before enrolling them for the study.

1. Average pain intensity will be assessed using the Numerical Pain Rating (NPR) over the past week $\geq 2$ on a $0-10$ numerical pain scale.

2. Roland Morris Disability Questionnaire score $\geq 4^{26}$.

3. Fear Avoidance Beliefs Questionnaire (FABQ) work subscale score $<1927$.

\section{Exclusion Criteria}

To be eligible for the study, participants must not:

- Have a personal history of the following neurological disorders: Alzheimer's, 
Amyotrophic Lateral Sclerosis, Multiple Sclerosis, Parkinson's, Stroke

- Have a personal history of the following cardiorespiratory disorders: Congestive heart failure, Heart attack in past 24 months

- Have a personal history of the following musculoskeletal disorders: Rheumatoid arthritis, Pathologic fractures of the spine, avascular necrosis or osteonecrosis, severe osteoarthritis. Including a history of spine surgery or a hip arthroplasty.

- Have active cancer.

- Be Blind.

- Have used narcotics or muscle relaxants within 30 days before study enrolment.

- Report being pregnant, lactating, or that they anticipate becoming pregnant in the next 3-6 months.

- Have a body mass index greater than 35 $\mathrm{kg} / \mathrm{m}^{2}$.

- Have clinical depression (i.e., subjects who score 24 or higher on the Center for Epidemiology Depression Scale).

- Report unexplained weight loss over the past month (>10 lbs.).

\section{Interventions}

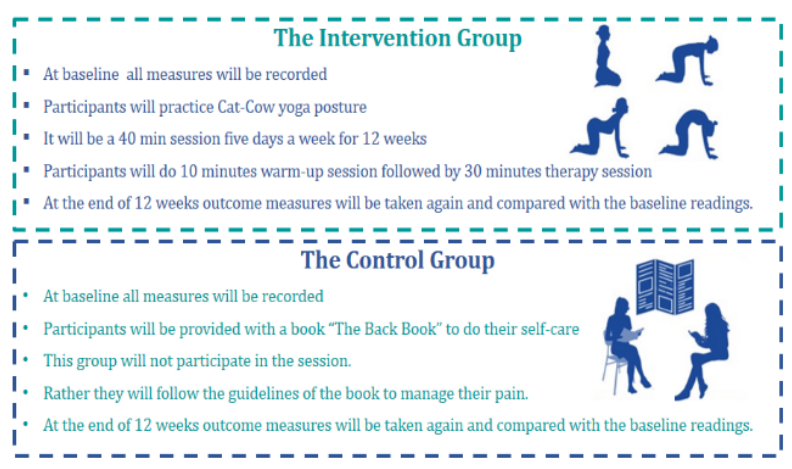

\section{Study Procedure}

Enrolment: Those subjects who had experienced musculoskeletal CLBP were enrolled.

Assessment of eligibility: Subject meeting eligibility criteria will be included in the study.
Baseline assessment: All the variables, i.e., Oswestry Low Back Pain Disability Questionnaire, World Health Organization Quality of life (WHOQOL) Questionnaire, Numerical Pain Rating Scale, Sadaf Stress Scale (SSS) will be measured at baseline.

Scoring of Oswestry Scale:

- $0 \%$ to $20 \%$ : minimal disability: The patient can cope with most living activities. Usually, no treatment is indicated apart from advice on lifting, sitting, and exercise.

- 21\%-40\%: moderate disability: The patient experiences more pain and difficulty sitting, lifting, and standing. Travel and social life are more difficult, and they may be disabled from work. Personal care, sexual activity, and sleeping are not grossly affected, and the patient can usually be managed by conservative means.

- 41\%-60\%: severe disability: Pain remains the main problem in this group, but activities of daily living are affected. These patients require a detailed investigation.

- 61\%-80\%: crippled: Back pain impinges on all aspects of the patient's life. Positive intervention is required.

- $81 \%-100 \%$ : These patients are either bedbound or exaggerating their symptoms ${ }^{28}$.

Randomization: Subjects based on eligibility criteria will be randomly allocated to the experimental or control group sequentially as they agree to participate.

Allocation: A booklet with detailed instructions will be provided to the study subjects according to the groups allocated.

Follow-up assessment: After three months, all the variables measured at the baseline phase will be measured again. 
Statistical Analysis: Pre \& post-analysis will be conducted in this phase.

\section{Expected Outcomes}

At the end of this study and proper execution of the intervention, it is expected that,

i. The subjects with musculoskeletal CLBP will overcome the pain and perform their daily tasks more efficiently.

ii. Levels of substance P and cortisol will be reduced, which will help to break the pain cycle in the body.

iii. Level of beta-endorphins will be elevated at the end of the session, which will make them relax and will help in further reduction of pain.

\section{Measures}

i. Oswestry Low Back Pain Disability Questionnaire: This index is considered a standard to assess the functionality of the low back. It is also a trusted tool for researchers and health care providers to evaluate the extent of disability ${ }^{29}$.

ii. Quality of life Questionnaire: It is used to measure the quality of life by various means. It will help evaluate changes observed in the patients' quality of life and well-being before and after the intervention is given ${ }^{30}$.

iii. Numerical Pain Rating Scale: To evaluate the intensity of pain.

iv. Roland Morris Disability Questionnaire: This scale is used to assess mild to moderate disability in patients with acute, subacute, or chronic low back pain ${ }^{26}$.

v. Sadaf Stress Scale (SSS): Sub-section of SSS (Physical Stress) will be used to assess the degree of stress and its contribution to CLBP 31 .

vi. Substance P: It gets increased in the body in chronic pain, so it could be a good source of estimating the extent of relief from the pain before and after the intervention. vii. Beta-Endorphins: They work as opioids in the body and produce endogenous analgesia. Their elevated levels after the intervention will be a sign of pain relief.

viii. Cortisol: It is a steroid hormone that tends to elevate during psychological or physical stress.

\section{Sample Size Calculation}

The required sample size for the two study groups with $\alpha=0.05$ and $(1-\alpha)=0.80$ was estimated to be 49 in each group, i.e., the total sample size was 98 for the two groups. The sample size calculator provided by the University of California, San Francisco (UCSF), Clinical and Translational Science Institute (CTSI) was used ${ }^{32}$.

\section{Randomization}

Subjects based imon eligibility criteria will be randomly allocated to the experimental or control group in the 1:1 ratio. Computergenerated random numbers will be used for randomization. After taking the subject's basic information, the study center will provide a unique code to each included subject. The code will be mentioned in each form of each subject.

\section{Sample Analysis}

All the biomarkers (Substance P, Beta Endorphins, Cortisol) will be analyzed and processed through ELISA.

\section{Statistical Analysis}

The data will be analyzed using $2 \times 2$ mixed factorial design analysis of variance (ANOVA) to calculate whether there is a significant change in the cat-cow yoga therapy group among the intervention and control group subjects. After the intervention, if a higher ratio of cat-cow yoga therapy will be observed in the experimental group, the interventional impact of the five factors of cat-cow yoga therapy will be examined with further analysis. Sequentially for each secondary outcome, additional 


\section{Annals of Psychophysiology $\quad$ ISSN 2412-3188 (Online) | $2410-1354$ (Print)}

ANOVAs will be used to investigate differences between groups at three months and baseline. Adjusted ANOVA will be performed, keeping socio-demographic and

other variables as co-variants to determine whether the socio-demographic and other characters could result in alterations in effect between the two groups.

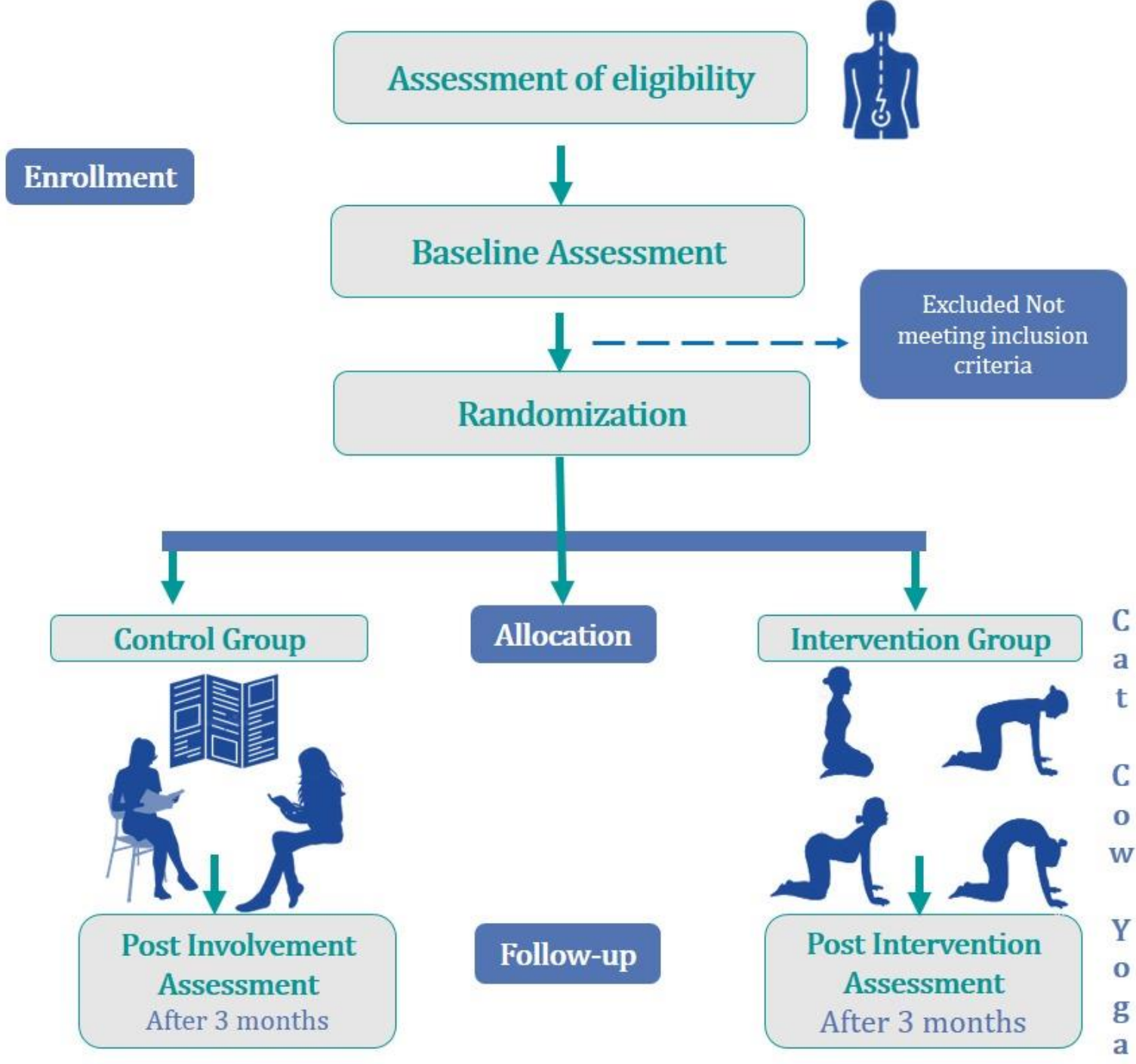

Figure 1: Flowchart of the study procedure

\section{Discussion}

The focus of research related to pain management is leaning towards all the interventions that could be used instead of pharmaceutical drugs and have the same efficacy and convenient for use. This study aims to find a correlation between psychophysiological biomarkers and CatCow yoga posture in the management of non-specific chronic low back pain. Substance P, beta endorphins, cortisol and stress levels are all going to be measured and monitored and on these basis efficacy of the intervention will be judged. Life-threatening events aside, people's economic statuses are subject to considerable effects. From purchasing high-cost medication to visiting healthcare advisors, this precarious situation 
will prove to be an inconvenience to people. Thus, an intervention - both, maximizing practicality and budget-friendliness - is needed. The topic field of this study, therefore, will revolve around the efficacy of a convenient and cost-effective yoga technique, fashioned to mitigate psychophysiological manifestations of CLBP.

\section{Acknowledgement}

The author is thankful to Arooma Zehra, Sukaina Reza and Khudaija Reza for their support throughout the process.

\section{References}

1. Zaina $F$, Balagué $F$, Battié $M$, Karppinen J, Negrini S. low back pain rehabilitation in 2020: new frontiers and old limits of our understanding. Eur J Phys Rehabil Med 2020;56:212-219.

2. Al-Arfaj AS, Al-Saleh SS, Alballa SR, AlDalaan AN, Bahabri SA, Al-Sekeit MA, Mousa MA. How common is back pain in AlQaseem region. Saudi Med J. 2003;24(2):170173.

3. Trinkoff AM, Lipscomb JA, Geiger-Brown J, Brady B. Musculoskeletal problems of the neck, shoulder, and back and functional consequences in nurses. Am J Ind Med. 2002;41(3):170-178.

4. Vos T, Flaxman AD, Naghavi M, Lozano R, Michaud C, Ezzati M, Shibuya K, Salomon JA, Abdalla S, Aboyans V, Abraham J. Years lived with disability (YLDs) for 1160 sequelae of 289 diseases and injuries 1990-2010: a systematic analysis for the Global Burden of Disease Study 2010. The lancet. 2012;380(9859):2163-2196.

5. Shieh SH, Sung FC, Su CH, Tsai Y, Hsieh VC. Increased low back pain risk in nurses with high workload for patient care: A questionnaire survey. Taiwan J Obstet Gynecol. 2016;55(4):525-529.

6. Jradi H, Alanazi H, Mohammad Y. Psychosocial and occupational factors associated with low back pain among nurses in Saudi Arabia. J Occup Health. 2020;62(1):e12126.
7. Dawson AP, Schluter PJ, Hodges PW, Stewart S, Turner C. Fear of movement, passive coping, manual handling, and severe or radiating pain increase the likelihood of sick leave due to low back pain. PAIN. 2011;152(7):1517-1524.

8. Ellapen TJ, Narsigan S. Work-related musculoskeletal disorders among Nurses: a systematic review. Ergonomics. 2014;4(4):1-6.

9. Tinubu BM, Mbada CE, Oyeyemi AL, Fabunmi AA. Work-related musculoskeletal disorders among nurses in Ibadan, Southwest Nigeria: a cross-sectional survey. BMC Musculoskelet Disord. 2010;11(12).

10. Elnaggar RK, Elshazly FA, Elsayed WS, Ahmed AS. Determinants and relative risks of low back pain among the employees in AlKharj area, Saudi Arabia. Eur J Sci Res. 2015;135(3):299-308.

11. Al Amer HS. Low back pain prevalence and risk factors among health workers in Saudi Arabia: A systematic review and metaanalysis. J Occup Health. 2020;62(1):e12155.

12. Alzahrani H, Mackey M, Stamatakis E, Zadro JR, Shirley D. The association between physical activity and low back pain: a systematic review and meta-analysis of observational studies. Sci Rep. 2019;9(8244):110.

13. Kasa AS, Workineh Y, Ayalew E, Temesgen WA. Low back pain among nurses working in clinical settings of Africa: systematic review and meta-analysis of 19 years of studies. BMC Musculoskel Dis. 2020;21(1):1-1.

14. Rasheed H, Rashid J \& Javeed RS. Frequency of low back pain among nurses working in Jinnah hospital Lahore. IJEHSR. 2017;5(3):4451.

15. Lisowska B, Lisowski A, Siewruk K. Substance $\mathrm{P}$ and chronic pain in patients with chronic inflammation of connective tissue. PloS one. 2015;10(10):e0139206.

16.Zieglgänsberger W. Substance $P$ and pain chronicity. Cell Tissue Res. 2019;375:227-241.

17. Snijdelaar DG, Dirksen R, Slappendel R \& Crul BJP. Substance P. Europ J Pain. 2000;4:121-135.

18. Sprouse-Blum AS, Smith G, Sugai D, Parsa FD. Understanding endorphins and their importance in pain management. Hawaii Med J. 2010;69(3):70. 
19. Ferdousi M, Finn DP. Stress-induced modulation of pain: role of the endogenous opioid system. Progress Brain Res. 2018;239:121-177.

20. Kallman TF, Ghafouri B, Bäckryd E. Salivary beta-endorphin and substance $\mathrm{P}$ are not biomarkers of neuropathic chronic pain propensity. Heliyon. 2018;4(8):e00718.

21. Almeida M, Saragiotto B, Richards B, Maher CG. Primary Care Management of NonSpecific Low Back Pain: Key Messages from Recent Clinical Guidelines. Med J Aust. 2018;208(6):272-275.

22. Shipton EA. Physical therapy approaches in the treatment of low back pain. Pain Ther. 2018;7(2):127-137.

23. Kamper SJ, Yamato TP, Williams CM. The prevalence, risk factors, prognosis and treatment for back pain in children and adolescents: an overview of systematic reviews. Best Pract Res Clin Rheumatol. 2016;30(6):1021-1036.

24. Wieland LS, Skoetz N, Pilkington K, Vempati $\mathrm{R}$, D'Adamo CR, Berman BM. Yoga treatment for chronic non-specific low back pain. Cochrane Database of Systematic Reviews. 2017(1).

25. Tsai SY. Effect of Yoga Exercise on Premenstrual Symptoms among Female Employees in Taiwan. Int J Environ Res. Pub Health. 2016;13(7).

26. Roland M, Waddell G, Klaber-Moffett J, Burton A, Main C. The Back Book: the best way to deal with back pain. 2nd ed. TSO. 2002:1-25.

27. Waddell G, Newton M, Henderson I, Somerville D, and Main C. A Fear-Avoidance Beliefs Questionnaire (FABQ) and the role of fear-avoidance beliefs in chronic low back pain and disability. Pain. 1993;52:157-168.

28. Roland M, Wadell G, Moffett J K, Burton K, Main C, Cantrell T. The Back Book. UK. The Stationery Office. 1996. Available at: https://backaholic.files.wordpress.com/201 4/12/the_back_book-2.pdf

29. Fairbank JCT \& Pynsent, PB. The Oswestry Disability Index. Spine. 2000;25(22):29402953

30. World Health Organization. (2004). The World Health Organization quality of life (WHOQOL) - BREF, 2012 revision. World Health Organization. Available at: https://apps.who.int/iris/handle/10665/7 7773

31. Ahmed S \& Noushad S. Sadaf Stress Scale; Reviewed Version tested on Pakistani Population. APP. 2015;4:44-47.

32. Kohn MA, Senyak J. Sample Size Calculators. UCSF CTSI. 2020;2020. Available at: https://www.sample-size.net/.

\section{Check for updates}

